\title{
Valutare senza voto numerico: strumenti e riflessioni di una sperimentazione di matematica alle scuole secondarie di primo grado
}

\author{
Assessment without numerical grades: \\ tools for and reflection on an alternative method \\ in mathematics for lower secondary school
}

Francesco D’Intino
Istituto Comprensivo Dante Alighieri - Rimini, Italia

Sunto / Il contributo presenta i passi di una sperimentazione, condotta nell'arco di due anni in una scuola secondaria di primo grado della provincia di Rimini, nella quale si è scelto di fare a meno del voto numerico in decimi. Dopo aver focalizzato i principali aspetti legati al tema della valutazione, vengono presentati alcuni dispositivi valutativi adottati nel primo anno di sperimentazione al fine di potenziare una visione formativa della valutazione; attraverso una raccolta dati intermedia, si argomentano i motivi dei cambiamenti apportati per il secondo anno di sperimentazione. Viene poi presentata un'analisi qualitativa dell'esperienza, effettuata sulla base di un questionario di inizio e fine anno proposto agli studenti. Vengono infine proposte alcune conclusioni in termini di percezioni degli studenti e dell'insegnante, al fine di guidare la riflessione per gli anni a venire.

Parole chiave: valutazione in decimi; valutazione formativa; scuola secondaria di primo grado.

\begin{abstract}
This article traces the steps taken to design and evaluate an innovative assessment method which does not use a 1-10 grading scale. The research was conducted in a lower secondary school in Rimini over a two-year period. First, the main components of assessment are outlined, followed by a description of some assessment tools used during the first year of research, aimed at enhancing the formative function of assessment. It is then explained how data that was collected during the first year led to some modifications of the strategies adopted in the second year. A quantitative analysis is then carried out on the basis of two questionnaires completed by the students, one at the beginning and one at the end of the school year. Finally, conclusions are drawn about student and teacher perception, in order to inform a reflection for the coming years.
\end{abstract}

Keywords: 1-10 grading scale; formative assessment; lower secondary school.

Pur essendo uno dei fattori chiave del processo di insegnamento/apprendimento, il tema della valutazione è spesso oggetto di confusione. A questo riguardo, è utile proporre una classificazione dei vari tipi di valutazione che esistono:

1. «Valutazione predittiva (orientativa): ha lo scopo di prevedere le caratteristiche del percorso formativo più adatto a un soggetto (ad esempio i test di orientamento).

2. Valutazione diagnostica: si colloca nella fase iniziale del processo formativo e ha lo scopo di analizzare le caratteristiche in ingresso di un allievo in relazione al percorso che dovrà compiere.

3. Valutazione formativa: ha lo scopo di fornire un feedback all'allievo e all'insegnante. 
4. Valutazione sommativa: si colloca alla fase conclusiva di un percorso formativo (modulo didattico, anno scolastico ecc.) e ha lo scopo di verificare i risultati conseguiti dall'allievo.

5. Valutazione certificativa: ha lo scopo di attestare socialmente i risultati da parte del soggetto in funzione delle sue scelte successive o dell'inserimento nel mondo del lavoro».

(Castoldi, 2012, pp. 163-164)

Come emerge da queste definizioni c'è una varietà molto vasta di significati; in questo contributo ci si soffermerà soprattutto sulla valutazione formativa e sommativa. La scelta di concentrare l'attenzione su questi due tipi di valutazione è motivata da due fattori: in primo luogo, le due modalità valutative sono le più frequenti nella quotidianità della didattica; in secondo luogo, spesso vi è il rischio di confonderle tra loro attraverso I'utilizzo di un unico strumento valutativo, il voto numerico. A tal proposito, scrive Capperucci (2011):

«Mentre in passato l'attenzione era rivolta principalmente a temi connessi con
il "prodotto" dell'evento formativo, oggi l'implementazione delle pratiche
valutative risponde all'esigenza di misurare l'efficacia e l'impatto esterno
delle singole azioni formative, e per questo è necessario sottoporre il sistema
formativo a indagini relative sia agli aspetti processuali che ai risultati prodotti».

(Capperucci, 2011, p. 149)

Nonostante le due valutazioni abbiano significati e strumenti operativi diversi fra loro, I'utilizzo pressoché esclusivo, nella pratica didattica italiana, di una valutazione di tipo sommativo rischia di oscurare la valenza di molte pratiche di valutazione formativa. Eppure sono anni ormai che la letteratura pedagogica e didattica di riferimento sostiene che «la valutazione dovrebbe servire innanzitutto a migliorare l'apprendimento» (Dozio, 2011, p. 10), identificando nelle pratiche di valutazione formativa alcuni elementi chiave per favorirlo. D'altro canto, una valutazione sommativa che utilizza il voto numerico come risposta alla prova dello studente può rendere difficoltosa una lettura utile di ciò che è stato verificato e creare molte problematiche. Di seguito, se ne elencano alcune.

- Eccessiva semplificazione: il risultato della prova è riassunto da un unico voto; studenti diversi con lo stesso voto possono evidenziare difficoltà totalmente differenti l'uno dall'altro; è il risultato di una prestazione senza accennare al processo che ha portato a essa.

- Mancanza di progressione: il voto numerico nasconde la progressione degli apprendimenti perché nelle varie prove svolte durante l'anno vengono valutati apprendimenti diversi.

- Deresponsabilizzazione: I'eccessiva semplificazione del voto può indurre nello studente l'idea di non poter agire in modo attivo sul risultato perciò non si sente stimolato a migliorare.

- Falsa scientificità: il voto numerico può dare l'idea di oggettività e univocità ma in realtà non esprimendo una misura fisica porta con sé una componente soggettiva.

- Priorità della performance: lo studente si concentra soltanto sul risultato finale, lo studio è impostato unicamente sull'esito (numerico) della prova.

- Ansia da prestazione: I'importanza della prestazione può causare difficoltà emotive che non consentono all'alunno di dare il meglio di sé. 
- Personalizzazione del giudizio: non esistendo una descrizione approfondita della performance, il voto può essere vissuto come un giudizio sulla persona.

- Condizionamento negativo della comunicazione: studenti e genitori si focalizzano sui voti senza considerare altri aspetti.

- Distorsione del ruolo dell'errore: I'errore viene vissuto e visto come qualcosa da evitare perché causa un abbassamento del voto; lo studente tende a nascondere le difficoltà all'insegnante.

- Comportamenti scorretti: se il fine è il voto, tutto può diventare lecito durante la prova, anche le scorrettezze.

- Competizione insana: il momento della consegna dei risultati può essere vissuto come una gara a chi ottiene il voto più alto.

- Penalizzazione ingiusta: spesso il voto numerico va a incidere in particolare sugli studenti di ambiente socioculturale svantaggiato.

In aggiunta a ciò, Castoldi (2012), approfondisce la riflessione sulla valutazione compilando un elenco di luoghi comuni su di essa dei quali evidenzia gli aspetti non veritieri. Ad esempio, un'immagine falsata dell'oggettività della valutazione, che non tiene conto di quanto il processo valutativo si fondi su una specifica e soggettiva rappresentazione della realtà da parte del valutatore. Oppure la diffusa idea secondo la quale la valutazione serva a sanzionare, senza tener conto degli aspetti formativi. O ancora:

«La valutazione in voti è più chiara: più che di chiarezza è più corretto parlare di maggiore familiarità con il giudizio in voti (immediata lettura, facile comparazione, si riescono a trattare più valutazioni insieme attraverso la media). La scala dei nostri giudizi è però ordinale quindi, indicando una posizione nella graduatoria, perdono il senso sia la differenza tra i giudizi che la media. [...] II giudizio come media dei voti: è un'operazione tecnicamente scorretta e professionalmente pericolosa; un presupposto logico imprescindibile dall'utilizzo della media è di avere elementi equivalenti, cosa che invece non si verifica né in una stessa materia (le verifiche possono avere gradi di difficoltà diversi oppure riguardare obiettivi differenti) né in più materie (non si può ritenere equivalente il giudizio in matematica e quello in inglese o quello nel comportamento). Sul piano professionale la media dei giudizi invece risulta pericolosa perché confonde il momento di rilevazione e quello di giudizio, quest'ultimo infatti si deve basare su un apprezzamento complessivo e globale dei dati e delle informazioni raccolti nel primo momento».

(Castoldi, 2012, p. 141)

Anche Bottero (2014) si concentra sulla definizione del termine valutazione, il cui significato è «formulare un giudizio sulla qualità di un'azione formativa» (Bottero, 2014, p. 3), e coinvolge il concetto di apprezzamento dei dati raccolti in una prova. L'insegnante non ha strumenti per misurare l'esito di una prova se non quello di avanzare un'interpretazione di ciò che è stato svolto dagli studenti e che, in quanto tale, non può considerarsi oggettiva ma porta con sé una chiave di lettura estremamente soggettiva.

Ma se la valutazione non può essere completamente oggettiva, non rimane altro obiettivo che «essere equi, credibili e di saper chiarire le scelte che hanno portato alla formulazione di un certo giudizio» (Dozio, 2011, p. 29). Detto in altri termini, il compito dell'insegnante riguardo alla valutazione è di confrontare ciò che è osservabile 
in ogni prova con quanto si era aspettato di rilevare; valutare significherà per il docente esprimere il grado di soddisfazione circa l'apprendimento dello studente alla fine di un percorso. Per rendere operativo questo tipo di obiettivo, Castoldi (2012, p. 147) propone un ciclo di elementi che riassumono il processo di valutazione:

1. Individuazione dell'oggetto: bisogna avere chiaro cosa si vuole valutare; si può fare riferimento alla dimensione cognitiva, che riguarda conoscenze e abilità, oppure anche a quella extra-cognitiva, che include aspetti emotivi, sociali e metacognitivi.

2. Definizione dei criteri: la domanda guida si formula in base a cosa si vuole valutare; è ciò che lega il momento progettuale a quello valutativo. Oltre ai criteri si possono stabilire le rispettive incidenze durante la valutazione. Si deve definire anche la modalità di attribuzione del giudizio all'allievo che può fare riferimento a una prestazione ritenuta ottimale, dipendere dal confronto tra la prestazione del singolo e del gruppo oppure apprezzare il progresso manifestato dall'allievo rispetto al suo livello iniziale.

3. Espressione del giudizio: l'insegnante deve scegliere quale tipo di codice usare per l'espressione del giudizio; può indicare la presenza o assenza di una certa condizione (nominale), può servirsi di un certo numero di livelli (ordinale) oppure può quantificare la prestazione facendo riferimento a un'unità di misura (metrico).

4. Regolazione dell'insegnamento: la valutazione riguarda non solo l'allievo ma anche l'insegnante che riceve interessanti feedback dalle prove dei suoi studenti che lo aiuteranno a riconoscere l'efficacia di quanto svolto in classe.

5. Comunicazione del giudizio: è necessario prevedere una modalità di comunicazione che si adatti ai vari utenti destinatari che possono essere gli studenti stessi, i loro genitori oppure altri docenti.

6. Ruoli dei soggetti: è importante stabilire con chiarezza chi valuta, lavorando su una dimensione orientata sulla collegialità; anche lo studente ha un suo ruolo nella valutazione infatti se ne deve servire affinché lo aiuti nell'apprendimento.

7. Requisiti di qualità: la valutazione deve essere attendibile, trasparente, utile e condivisa.

Quanto appena enunciato orienta il discorso su una valutazione di tipo criteriale, in cui devono essere chiari, sin dall'inizio e a tutti i soggetti coinvolti, gli obiettivi da raggiungere in un certo periodo di tempo. Anche i criteri valutativi devono essere stabiliti con giusto anticipo affinché possano guidare la didattica e orientare gli studenti durante l'apprendimento. Oltre a una programmazione attenta e ragionata, ai docenti viene richiesta anche una chiarezza nell'informazione, che viene influenzata dai destinatari; ' 'insegnante infatti sarà costretto a modificare la modalità di comunicazione a seconda che stia parlando con i colleghi, gli studenti o i genitori.

Ad esempio, in un colloquio con i genitori sarebbe inefficace mostrare esclusivamente i voti numerici ottenuti dallo studente senza prima aver fornito elementi descrittivi che siano illustrativi del lavoro svolto fino a quel momento. Uno scambio informativo completo, articolato, argomentato e adeguato alle situazioni potrebbe invece favorire una comprensione profonda della situazione e aiutare tutte le parti coinvolte a migliorare il proprio apporto, ciascuno secondo le sue finalità. 


\subsection{Valutazione e apprendimento}

Secondo Dozio (2011), la valutazione:

«[...] dovrebbe servire innanzitutto a migliorare l'apprendimento; è questo il vero scopo dell'osservazione del lavoro dell'allievo e del nostro valutare la situazione dell'allievo in relazione agli obiettivi da raggiungere. Osservare e valutare per dare indicazioni all'allievo sul prossimo lavoro di apprendimento da affrontare».

(Dozio, 2011, p. 10)

In particolare la valutazione formativa accompagna lo studente durante l'acquisizione delle competenze e lo aiuta a esaminare le proprie strategie e a migliorarle per raggiungere il successo formativo; proprio in questo senso si rivela una valutazione per l'apprendimento, differenziandosi da quella sommativa che invece è una valutazione dell'apprendimento.

Per fare in modo che ogni studente abbia la possibilità di riflettere sul proprio operato, sfruttando la valutazione per migliorare il proprio apprendimento, è dunque necessario che questa si trasformi in uno strumento a disposizione degli studenti. D'altro canto, questa trasformazione sarà utile anche all'insegnante, che se ne potrà servire per:

- comprendere più dettagliatamente le difficoltà disciplinari degli studenti;

- trarre indicazioni utili a sostenere al meglio i propri studenti e per guidare la progettazione didattica;

- riflettere sulla disciplina e sulle proprie convinzioni didattiche col fine di regolare l'insegnamento;

- rendere sempre più chiaro a se stesso e agli studenti le caratteristiche del proprio metodo valutativo.

Di cosa necessita la valutazione per diventare una valutazione per l'apprendimento? Un punto importante è quello riguardante i criteri di valutazione: una loro descrizione approfondita permette di ottenere una base solida sulla quale è possibile instaurare un dialogo chiaro tra chi valuta e chi apprende. La co-costruzione in termini di obiettivi di questi criteri insieme agli studenti, poi, permette all'insegnante di suscitare l'attenzione dello studente con indicazioni formative specifiche riguardanti le difficoltà evidenziate; da ciò può nascere un confronto proficuo che avrà come punto di partenza l'autovalutazione dell'allievo riguardo alla propria prova e il confronto con la valutazione ottenuta, obbligandolo a rivedere quanto ha prodotto. I benefici di questa pratica potrebbero essere dunque molteplici:

- spostare l'oggetto dell'attenzione dello studente, affinché la valutazione della prova non venga avvertita come un giudizio alla persona ma come indicatore di un livello di apprendimento;

- rendere la valutazione facilmente comprensibile e profondamente descrittiva in ogni suo aspetto, evitando fenomeni di appiattimento e non significatività della valutazione;

- contrastare il disinteresse e la paura dell'errore, veicolando invece l'idea che le negatività emerse da una prova devono essere accettate, conosciute e sfruttate per migliorare l'apprendimento.

Una valutazione chiara nei criteri, che frammenta l'oggetto di apprendimento in obiettivi più piccoli, può inoltre aiutare gli studenti ad acquisire un maggiore senso di autoefficacia e una minore passività, stimolando la loro motivazione ad apprendere. È chiaro che intendere la valutazione in questo modo richiede anche un ruolo più attivo da parte degli studenti, che svilupperanno un maggiore controllo sul proprio 
processo di apprendimento e attiveranno quelle risorse metacognitive che consentono l'autoriflessione e l'autovalutazione.

Questo cambio di prospettiva indirizza lo studente verso una riflessione profonda e potrebbe permettergli di essere più attento a ciò che vive a scuola, sia in termini predittivi, cioè nella fase di studio prima della prova, che riflessivi, cioè dopo la prova nella rilettura degli errori e nel dialogo con il docente.

La maggiore partecipazione attiva dello studente, le strategie autovalutative e l'autoregolazione, intesa come farsi carico dei propri processi cognitivi e motivazionali per raggiungere un obiettivo, sono tutte caratteristiche metacognitive irrinunciabili per una scuola che miri alla crescita formativa dei suoi studenti.

Se è vero che la scuola «non ha più lo scopo di identificare una élite sociale destinata a proseguire gli studi, ma di formare nel miglior modo possibile un numero massimo di allievi» (Dozio, 2011, p. 5) allora questo obiettivo è una sfida per la scuola dell'obbligo, nella quale può giocare un ruolo importante la valutazione intesa come processo complesso nel quale intervengono vari attori, strumenti, conoscenze e competenze inerenti al processo di insegnamento/apprendimento.

\section{2}

\section{Il contesto della sperimentazione}

La sperimentazione descritta in questo contributo ha avuto luogo in tre classi, una prima, una seconda e una terza, della scuola secondaria di primo grado ${ }^{1}$ dell'istituto comprensivo Dante Alighieri di Rimini.

L'accoglienza a livello di contesto e la qualità delle relazioni fra colleghi promuovevano un clima adatto alla sperimentazione.

Dopo un colloquio con la dirigente, a settembre 2017, è iniziata una fase di documentazione di sperimentazioni analoghe a quella che si intendeva progettare. La conversazione con la dirigente ha sollecitato la riflessione circa l'impatto che la proposta di cambiamento nella valutazione avrebbe creato. Pensando alla sperimentazione, le principali problematiche che interessavano il contesto riguardavano in primo luogo la comunicazione ai genitori degli studenti: non comunicare la valutazione in decimi avrebbe potuto essere una complicazione nel caso di una non promozione alla fine dell'anno; la famiglia infatti avrebbe potuto sostenere di non essere stata in grado, attraverso i giudizi scritti dell'insegnante, di capire la situazione gravemente insufficiente del figlio. Anche i genitori, inoltre, sono solitamente abituati al voto e quindi sarebbe stato necessario descrivere dettagliatamente le scelte didattiche nelle riunioni di inizio anno. L'ultima incertezza riguardava la mancanza di affinità con il metodo valutativo degli altri colleghi del consiglio di classe; una diversità tale avrebbe potuto avere conseguenze sia tra i colleghi che con gli studenti e le rispettive famiglie.

\subsection{Prime esplorazioni}

L'anno scolastico 2017/2018 è stato l'occasione da un lato per sperimentare alcune strategie e strumenti al fine di poter proporre un progetto basato su dati, dall'altro

1. La scuola secondaria di primo grado in Italia dura tre anni e corrisponde ai primi tre anni di scuola media nel Canton Ticino. 
per documentarsi su alcuni modelli valutativi già attivati in differenti contesti al fine

di integrarli con altre scelte che mirano alla riflessione personale di ogni studente sul proprio operato.

La proposta per l'anno 2017/2018 è stata di attivare alcuni dispositivi di autovalutazione, integrati ad altri dispositivi di valutazione utilizzati dal docente. In aggiunta a queste due categorie, è stato proposto agli studenti un questionario di fine anno il cui scopo era indagare il corretto utilizzo dei dispositivi di autovalutazione attivati e le percezioni circa il nuovo metodo di valutazione.

\title{
2.1.1 Dispositivi di autovalutazione per gli studenti
}

\author{
Commento a caldo.
}

Al termine di ogni verifica scritta, gli studenti scrivono un commento riguardo alla prova appena sostenuta; non è un compito strutturato e può riguardare il loro grado di soddisfazione circa la prova appena svolta, se pensano che il docente li abbia preparati in modo sufficiente, se la prova era troppo lunga, se si sentivano pronti o se ritenevano di aver studiato in modo adeguato. Questo commento scritto di getto, appena terminata la verifica, ha la funzione di sollecitare un breve momento riflessivo per fissare le prime impressioni, favorendo il ricordo vivo di quanto vissuto durante la prova; permette appunto di fare memoria e di avere indicazioni su tanti aspetti anche molto diversi tra loro: stato emotivo dell'alunno, opinioni sulla matematica, opinioni su se stessi e su come lavorano, opinioni sulle loro attese, opinioni sull'operato dell'insegnante, opinioni sulla verifica.

\section{Riflessione finale.}

All'indomani della correzione della verifica, lo studente scrive sul proprio quaderno un breve testo che tenga conto delle aspettative antecedenti alla verifica, degli errori commessi nella prova e anche della correzione fatta insieme in classe; è una sorta di relazione finale che deve soppesare le aspettative e le considerazioni presenti nel commento a caldo, per poi confrontarle con il giudizio e i commenti ricevuti dall'insegnante (si veda il par. 2.1.2). Grazie a questo strumento, ogni studente è nella condizione di riflettere sulla propria prova in due diversi momenti temporali, ognuno dei quali contraddistinto da un numero crescente di informazioni; I'obiettivo è di aiutarli a leggere ciò che è successo con un grado sempre maggiore di profondità,

\footnotetext{
2. Ad esempio, il modello della Scuola del gratuito, progetto della Comunità Papa Giovanni XXIII, in cui la sperimentazione del prof. Ciani $(2008,2013)$ prevede la responsabilizzazione dei ragazzi nelle scelte didattiche e di comportamento in classe; riguardo alla valutazione invece il voto numerico è accompagnato da una lettera rivolta allo studente nella quale vengono analizzate le positività emerse dalla prova. Allo studente spetta la decisione di conoscere o meno il voto in decimi ottenuto. Per maggiori informazioni, è possibile consultare il link: https://scuoladelgratuito.wordpress.com/about/.

In una scuola primaria di Novara, Davide Tamagnini (2019) ha sostituito il voto numerico con una tabella in cui vengono elencate le diverse abilità, conoscenze, competenze e per ciascuna viene associato un colore (verde, giallo o rosso) a seconda che esse siano raggiunte, in lavorazione o presentino delle evidenti difficoltà. Per maggiori informazioni sul Movimento di Cooperazione Educativa, è possibile consultare il link: http://www.mce-fimem.it/.

Nella scuola primaria "Collodi" di Pisa è stato eliminato il voto numerico a favore di strumenti di carattere narrativo, adatti a definire i reali punti di forza e di debolezza di ciascun alunno in modo da indicare loro dei precisi percorsi di crescita e di miglioramento a seconda delle specifiche necessità. Per maggiori informazioni, consultare il link: http://moodle.mce-fimem.it/pluginfile.php/4611/mod_resource/content/3/ Valutazione\%20Senza\%20Voto\%20Gabriele\%20Recchia.pdf.

Un'altra esperienza è la Classe senza voto (Bacchi \& Romagnoli, 2019), sperimentata presso il liceo Monti di Cesena, in cui nella valutazione formativa viene sostituito il voto numerico con una valutazione per competenze, espresse su tre livelli di acquisizione.
} 
abituandoli col tempo a prevedere con più precisione quello che potrebbe essere l'esito di una prova.

File "Le mie prove".

Per ogni verifica scritta lo studente dovrà ricopiare su un file di testo il commento a caldo, il giudizio e i consigli del docente e la riflessione finale; il tutto deve essere condiviso su Google Drive, affinché il docente possa leggere le produzioni e avere informazioni aggiuntive sul percorso personale dello studente dal punto di vista metacognitivo. Questa memoria storica può favorire una conoscenza più approfondita dell'evoluzione dell'apprendimento e dei processi di controllo attivati dai singoli studenti.

\subsubsection{Dispositivi di valutazione del docente} Giudizio in positivo.

È scritto dal docente e viene consegnato allo studente al momento della restituzione della verifica; ha il compito di analizzare, alla luce delle Indicazioni Nazionali italiane (MIUR, 2012), solamente gli obiettivi di apprendimento che sono stati raggiunti, valorizzando tutto ciò che lo studente ha mostrato di aver appreso. In questo modo si indebolisce la povertà descrittiva del voto numerico, rendendo più chiaro l'esito della prova scritta; inoltre questo strumento ha il pregio di focalizzare l'attenzione solamente sui contenuti della materia, disincentivando dunque una visione del voto come giudizio sulla persona (alcuni esempi di giudizi in positivo possono essere letti in Allegato 1).

Consigli.

Sono scritti dal docente allo scopo di accompagnare il giudizio in positivo evidenziando alcune difficoltà di tipo disciplinare e focalizzando l'attenzione sugli aspetti su cui poter migliorare; I'intento è di favorire un processo di riorganizzazione della propria conoscenza, individuabile ad esempio attraverso maggiori livelli di partecipazione e attenzione nei confronti della correzione della prova proposta alla lavagna dall'insegnante; questo strumento favorisce inoltre la possibilità di concentrarsi sugli errori commessi e sugli aspetti da migliorare; la redazione dei consigli, poi, è strumento di approfondimento dettagliato anche per l'insegnante, che può ad esempio riassumere in una tabella statistica facilmente consultabile quegli argomenti che hanno creato maggiori difficoltà durante la prova (alcuni esempi di consigli possono essere letti in Allegato 1). Voto numerico differito.

Il voto numerico viene specificato agli studenti qualche giorno dopo la correzione della prova. Questo strumento disincentiva un confronto competitivo non sano e il desiderio a volte ossessivo di conoscere il proprio voto e quello dei compagni. Inoltre, lo studente è incentivato a mettere in relazione l'andamento della prova a livello di giudizio scritto con il voto numerico ricevuto, giustificando una tale valutazione con le numerose informazioni aggiuntive che ha ottenuto dallo studio approfondito della verifica e delle difficoltà emerse.

\subsection{Problematiche emerse in corso d'anno}

Dopo aver comunicato e discusso insieme agli studenti riguardo al metodo di valutazione che si voleva implementare, sono stati utilizzati gli strumenti ipotizzati per un semestre intero. Con il passare dei mesi, sono emerse alcune problematiche:

- Giudizio in positivo. La sua scrittura richiede un onere di tempo eccessivo in quanto, per ogni argomento, è necessario trovare l'aggettivo giusto per descriverne la qualità dell'apprendimento raggiunta dallo studente; inoltre si è rivelato 
poco comprensibile per alcuni studenti, soprattutto quelli in difficoltà con la materia, che non riuscivano sempre a capire cosa avessero svolto in modo corretto.

- Voto numerico differito. Nel momento di riconsegna della prova, una buona parte di studenti si concentrava sul calcolo di punti fatti e punti totali, che segnalavo a fianco di ogni esercizio della verifica, e questo li distraeva totalmente dalla lettura del giudizio e dei consigli dell'insegnante. L'attesa del voto, inoltre, causava domande frequenti da parte di alcuni studenti nelle lezioni successive: ciò è indicativo di quanto lo ritenessero importante.

- File "Le mie prove". Non veniva compilato da tutti; inoltre, molti di quelli che lo facevano scrivevano in maniera piuttosto superficiale frasi troppo generiche e vaghe che non forniscono alcun tipo di informazione rilevante. Purtroppo pochi commenti a caldo e poche riflessioni finali apparivano significative e frutto di un serio impegno nella compilazione. Infine, dal punto di vista del docente, il controllo di questo compito si è rivelato piuttosto impegnativo e oneroso.

Proprio a causa delle difficoltà emerse, nella seconda parte dell'anno scolastico si sono apportate alcune modifiche agli strumenti ipotizzati, affinché gli studenti riuscissero a servirsene per gli scopi previsti. Si è deciso di fare a meno dei giudizi in positivo, sostituendoli con una struttura più schematica che risultasse di più facile compilazione e più fruibile. Se ne presentano di seguito le componenti.

Obiettivi di apprendimento e livelli.

La struttura di questo dispositivo è ispirata agli obiettivi di apprendimento presenti nelle Indicazioni Nazionali. Nelle ultime due verifiche dell'anno 2017/18, dopo gli esercizi e i problemi da svolgere, è stato inserito un elenco puntato con la lista degli obiettivi valutati all'interno della prova (in Allegato 2 si trova il dispositivo di valutazione declinato per le classi prima e seconda). Ad ognuno di questi obiettivi è stato associato un numero da 1 a 4, indicatore del livello raggiunto dallo studente nella prova; seguendo l'elenco puntato degli obiettivi, poi, è stato possibile numerare i consigli in modo puntuale e specifico, cosicché ogni studente potesse comprendere senza equivoci a quale obiettivo faceva riferimento ogni consiglio.

Lo scopo di questo dispositivo è di rendere più leggibile la valutazione e incentivare una maggiore consapevolezza negli studenti circa gli obiettivi verificati in ogni prova e il livello raggiunto per ognuno di essi, con relativi consigli. Questa modalità include anche il vantaggio di facilitare gli studenti nella riflessione e interiorizzazione degli obiettivi di apprendimento specifici della disciplina.

\subsection{Risultati del questionario di fine anno}

Il questionario finale era composto da due domande:

1. Quante volte hai completato il file "Le mie prove" su Google Drive, scrivendo il commento a caldo e la riflessione finale? [Sempre - Quasi sempre - Quasi mai Mai]

2. Pensi che una delle due modalità (giudizio in positivo oppure obiettivi di apprendimento e livelli) ti abbia aiutato nello studio della matematica? [Sì - No] Motiva la risposta in modo approfondito.

La prima domanda, a risposta chiusa, intendeva raccogliere dati riguardo all'utilizzo da parte degli studenti dei dispositivi di autovalutazione attivati durante l'anno. La seconda domanda era composta da due parti: una prima parte, a risposta chiusa, il cui intento era raccogliere un giudizio sintetico riguardo al nuovo metodo di valutazione utilizzato; una seconda parte, a risposta aperta, di motivazione del giudizio appena espresso. 
Valutare senza voto numerico: strumenti e riflessioni di una sperimentazione di matematica alle scuole secondarie di primo grado / Francesco D’Intino

Al questionario di fine anno hanno partecipato 55 studenti su 63, circa l'87\% del totale. In Figura 1 vengono riportati i risultati globali riferiti alla prima domanda.

\section{1) Quante volte hai completato il file "Le mie prove" su Google Drive, scrivendo il commento a caldo e la riflessione finale?}

Figura 1

Percentuale di risposte degli studenti alla prima domanda del questionario di fine anno.
Tabella 1

Motivazioni relative

alla risposta affermativa e negativa alla seconda domanda del questionario.

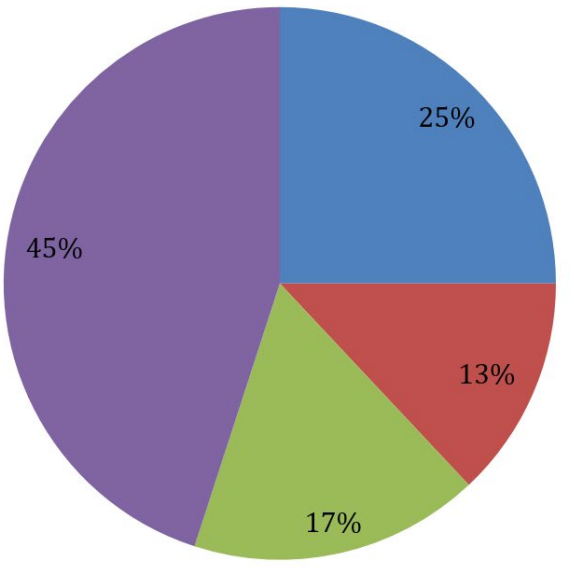

Mai

- Quasi mai

Quasi sempre

- Sempre

Poco più della metà degli studenti afferma di aver completato il file di verifica sempre o quasi sempre. Se si considera che lo strumento aveva lo scopo di stimolare l'autovalutazione e facilitare un lavoro metacognitivo sul proprio apprendimento, questo risultato non è soddisfacente. Tuttavia è comprensibile che, senza un controllo serio e costante da parte dell'insegnante, gli studenti abbiano prestato meno attenzione a questo obbligo che esula dai compiti solitamente richiesti a scuola.

Alla seconda domanda, con la quale si intendeva comprendere se gli alunni avessero considerato positivamente l'introduzione dei nuovi metodi valutativi, I'82\% degli studenti ha risposto in modo affermativo; il $18 \%$ ha invece dichiarato che il nuovo metodo valutativo non li ha aiutati nello studio della matematica. Le motivazioni per entrambe le categorie di risposta sono rappresentate in Tabella 1. Pensi che una delle due modalità (giudizio in positivo oppure obiettivi di apprendimento e livelli)
ti abbia aiutato nello studio della matematica? Motiva la risposta in modo approfondito.

\section{Motivazioni "Sì"}

\begin{tabular}{|l|l|l|l|}
\hline $\begin{array}{l}\text { Maggiore comprensione degli obiettivi } \\
\text { raggiunti e degli aspetti da migliorare }\end{array}$ & $54 \%$ & $\begin{array}{l}\text { Inutilità del nuovo metodo valutativo } \\
\text { rispetto al precedente }\end{array}$ & $5 \%$ \\
\hline Maggiore comprensione degli errori & $20 \%$ & Inefficacia dei consigli & $5 \%$ \\
\hline $\begin{array}{l}\text { Maggiore comprensione degli obiettivi } \\
\text { dello studio della matematica }\end{array}$ & $8 \%$ & Non so & $8 \%$ \\
\hline & $82 \%$ & & $18 \%$ \\
\hline
\end{tabular}

Dalla tabella si nota come la maggioranza degli studenti abbia tratto giovamento dalla sperimentazione di una delle due modalità valutative. Una buona parte degli 
studenti che ha risposto affermativamente sostiene che il metodo di valutazione li abbia aiutati a capire in quali obiettivi di apprendimento dovevano migliorare («Leggendo il commento capivo cosa dovevo migliorare e cosa so già fare bene», "Sono state entrambe molto di aiuto perché almeno sono riuscita a capire gli obiettivi che ho raggiunto di più e quelli di meno»), oppure a comprendere il ruolo dell'errore come opportunità ( QQuello con i livelli perché un livello mi aiuta a darmi un'autovalutazione e questo metodo mette più in evidenza quello che non va»), o ancora ad acquisire maggiore consapevolezza degli apprendimenti coinvolti nelle prove («Penso sia più costruttiva perché se sono chiari gli obiettivi per l'alunno è più semplice capire quali sono le aspettative e riesce a focalizzarsi di più sugli argomenti che il prof ritiene più importanti»).

In netta minoranza sono le risposte negative, nelle quali gli studenti sostengono di non aver tratto alcun vantaggio nello studio della matematica con il nuovo metodo valutativo. Alcuni di loro evidenziano come le informazioni siano tutte disponibili direttamente guardando la prova («Già dalla verifica vedo cosa migliorare»), altri ammettono di faticare nel tenere in considerazione i consigli forniti dal docente («Perché i consigli me li scordavo sempre»).

\section{Scelte per il secondo anno di sperimentazione}

Dall'analisi dei dati e dell'esperienza fatta durante I'anno scolastico 2017/2018, sono scaturite alcune riflessioni:

- in generale, l'utilizzo dei diversi dispositivi di valutazione e autovalutazione ha il pregio di essere più completo a livello descrittivo, aiutando gli studenti a capire come possono migliorare e il docente ad avere un quadro dettagliato per ciascuno studente;

- è necessario rendere più fruibile la griglia degli obiettivi utilizzata in fase di valutazione: gli studenti tendono a perdersi tra i numerosi obiettivi previsti dalle Indicazioni Nazionali e a non riuscire a fare memoria del proprio percorso di apprendimento; I'insegnante si trova costretto a rinumerare ogni volta i livelli raggiunti da ogni allievo distinguendoli per i relativi obiettivi;

- la lettura di dati numerici che indicano i livelli per ciascun obiettivo è molto più fruibile rispetto al giudizio e permette all'insegnante di avere un quadro abbastanza chiaro sul quale costruire e programmare la didattica delle lezioni successive alla verifica;

- diversi studenti avvertono ancora la necessità di confrontarsi con un voto numerico unico, grazie al quale potersi posizionare in modo sintetico rispetto al proprio andamento scolastico in matematica.

A partire da queste considerazioni, nella progettazione estiva l'attenzione si è concentrata sulla costruzione di una griglia valutativa di matematica che potesse essere condivisa con gli studenti e che diventasse per loro un mezzo efficace per riflettere sul proprio apprendimento e per avere allo stesso tempo indicazioni sintetiche riguardo agli esiti delle valutazioni.

Per questo si è deciso di utilizzare, in modo più preciso e puntuale rispetto a quanto fatto in precedenza, gli obiettivi di matematica previsti nelle Indicazioni Nazionali e di strutturare la griglia in modo che potesse valere per tutti gli anni scolastici e 
con la quale gli studenti, con il passare del tempo, potessero prendere sempre più confidenza ${ }^{3}$. Per fare questo si sono ripresi e numerati gli obiettivi di apprendimento di matematica, in modo da mostrare di volta in volta solo quelli presenti nelle varie prove di verifica. Per ognuno di questi, in linea con quanto fatto nell'ultima parte dell'anno precedente, si sarebbe fornito agli studenti il livello raggiunto in ogni prova; anche i consigli sarebbero stati numerati in riferimento agli obiettivi di apprendimento corrispondenti.

Questo lavoro di riadattamento degli obiettivi di apprendimento si è inserito in un ragionamento più ampio, nel quale si è ipotizzato il peso da dare a quattro diversi criteri di valutazione:

1. Obiettivi di apprendimento disciplinari (peso nella valutazione finale: $70 \%$ ): gli obiettivi indicati nelle Indicazioni Nazionali sono stati rielaborati e alcuni sono stati riscritti in maniera semplificata affinché la loro comprensione risultasse facile per tutti gli studenti; si è mantenuta la suddivisione nei quattro nuclei proposta nel documento (Numeri, Spazio e figure, Relazioni e funzioni, Dati e previsioni) (Allegato 3).

2. Comunicazione (peso nella valutazione finale: $10 \%$ ): non essendo presente in modo esplicito in nessuno dei 42 obiettivi previsti dalle Indicazioni Nazionali, si è scelto di valutare questo aspetto, considerando due indicatori: utilizzo del lessico specifico e capacità di organizzazione del discorso matematico.

3. Partecipazione (peso nella valutazione finale: $10 \%$ ): con questo criterio si vuole valutare la costanza degli studenti nella compilazione del file condiviso "Le mie prove", nell'esecuzione dei compiti assegnati e nell'attenzione ad avere sempre il materiale richiesto per il lavoro in classe.

4. Relazione (peso nella valutazione finale: $10 \%$ ): questo criterio invece comprende il comportamento che gli studenti tengono durante le lezioni, se sono collaborativi con i propri compagni e se rispettano l'insegnante e le regole di convivenza necessarie per lavorare insieme.

L'insieme di questi quattro criteri ha composto lo schema valutativo da applicare durante I'anno 2018/2019 (Allegato 4). Tutti gli obiettivi delle Indicazioni Nazionali sono stati numerati e inclusi nel primo criterio, in modo da essere facilmente riconoscibili in tutte e tre le classi. Al momento di una verifica scritta o di una prova orale, allo studente viene restituito un livello (da 1 a 4 ) per ogni obiettivo verificato. II secondo criterio può essere valutato in alcuni momenti specifici, sia attraverso prove scritte nelle quali si richiede di formulare spiegazioni di procedure matematiche generali, sia attraverso prove orali. Gli ultimi due criteri di valutazione sono oggetto di misurazione in modo discontinuo durante l'anno scolastico.

La suddivisione del primo criterio in obiettivi può aiutare gli studenti a capire cosa

3. Si è considerata la possibilità di fare riferimento ai traguardi per lo sviluppo delle competenze, sempre contenuti nelle Indicazioni Nazionali. Questi ultimi però presentano una caratteristica che si adatta poco a una valutazione ripetuta nel tempo in cui i contenuti cambiano e si ampliano man mano che si procede: i traguardi per lo sviluppo delle competenze sono infatti molto ampi e descrivono attese di apprendimento previste al termine della scuola secondaria di primo grado. La loro interpretazione può essere più complessa per gli studenti e impedisce loro di mettere a fuoco con precisione le difficoltà riscontrate nei vari aspetti matematici. Alcuni di essi inoltre rendono difficile una loro valutazione immediata, ad esempio: «L'alunno sostiene le proprie convinzioni, portando esempi e controesempi adeguati e utilizzando concatenazioni di affermazioni; accetta di cambiare opinione riconoscendo le conseguenze logiche di una argomentazione corretta» (MIUR, 2012, p. 63). Altri necessitano di un periodo lungo per poterne apprezzare i cambiamenti, ad esempio: "L'alunno ha rafforzato un atteggiamento positivo rispetto alla matematica attraverso esperienze significative e ha capito come gli strumenti matematici appresi siano utili in molte situazioni per operare nella realtà» (MIUR, 2012, p. 63). 
viene valutato in ogni prova e a fare memoria degli obiettivi che causano loro più difficoltà, così da poter riorganizzare lo studio e riflettere su di esso per migliorare nelle prove successive.

Per determinare il voto numerico al termine di ogni quadrimestre, come previsto dalla normativa scolastica, si è operato in questo modo:

- media tra le medie dei livelli raggiunti per ogni obiettivo;

- media tra le medie dei livelli ottenuti in lessico e in organizzazione del discorso;

- media dei livelli ottenuti nella partecipazione;

- media dei livelli ottenuti nella relazione.

Da queste quattro medie ottenute si procede effettuando una nuova media pesata, tenendo conto delle percentuali dichiarate nella griglia di valutazione (70\% obiettivi di apprendimento e $10 \%$ per i restanti criteri, cioè comunicazione, partecipazione e relazione); si va poi a confrontare il valore ottenuto con degli intervalli numerici decisi a priori (si veda l'Allegato 4, in fondo alla griglia di valutazione); a ogni intervallo viene associato il voto numerico corrispondente in decimi.

Si è deciso, infine, di definire il voto finale considerando tutti i livelli raggiunti durante l'anno scolastico; questa modalità fa sì che il voto conclusivo rappresenti tutto il percorso annuale, premiando chi si è impegnato e tenendo conto di chi è stato scostante. Fare una media tra molti dati inoltre ha il pregio di ammorbidire eventuali prestazioni eccezionali, positive o negative, che in tal modo pesano meno nella scelta del voto.

Ad inizio anno il metodo di valutazione deve essere spiegato in tutte le classi e deve essere consegnata una griglia cartacea che raccolga tutti i criteri, compresi tutti gli obiettivi di apprendimento, gli indicatori per ogni criterio e gli intervalli per assegnare il voto numerico alla fine di ogni quadrimestre. Tale griglia dovrà essere incollata sul quaderno e consultata con costanza da ogni studente.

Al fine di abituare gradualmente gli studenti e le famiglie all'assenza del voto numerico, si è anche scelto di esplicitare, per ogni valutazione scritta e orale, l'esito in termini di sufficienza o insufficienza. In questo modo gli studenti e i genitori hanno la possibilità di avere, in aggiunta a indicazioni analitiche e dettagliate, anche un'indicazione sintetica e globale del risultato di ciascuna prova.

L'insieme dei dispositivi di valutazione e autovalutazione da adottare durante l'anno sono dunque risultati i seguenti:

- commento a caldo;

- riflessione finale;

- file condiviso "Le mie prove";

- obiettivi e livelli; consigli;

- esito (sufficiente/insufficiente).

\subsection{Questionari (a.s. 2018/19)}

Al fine di ricevere informazioni utili riguardo alla percezione degli studenti riguardo ai metodi di valutazione implementati, si è scelto di far compilare loro un questionario sia a inizio che a fine anno (Allegato 5 e Allegato 6). II questionario di inizio anno consta di 13 affermazioni per le quali ogni studente deve indicare il livello di accordo o disaccordo sulla base di una scala Likert da 1 (per niente d'accordo) a 4 (completamente d'accordo). Le 13 affermazioni indagano le idee che gli studenti hanno riguardo al metodo di valutazione incentrato sul voto numerico e sono organizzate in 4 categorie: 
- voto numerico (affermazioni 1, 2) - il loro parere sul voto numerico;

- feedback (affermazioni 3, 4, 5, 6, 7) - quali informazioni ottengono dal metodo di valutazione;

- come migliorarsi (affermazioni 8, 9, 10) - in che modo sono supportati dal metodo di valutazione durante l'apprendimento;

- emotività (affermazioni 11, 12,13) - il loro stato d'animo nel rapporto con la valutazione.

Il questionario di fine anno comprende, come quello di inizio anno, 13 affermazioni incentrate sulle categorie sopracitate, alcune delle quali riguardano il metodo di valutazione basato su obiettivi, livelli e consigli. Nel questionario finale sono state aggiunte anche due domande, rielaborate a partire da quelle proposte l'anno precedente.

La prima domanda, a risposta chiusa, intende raccogliere dati riguardo all'utilizzo da parte degli studenti dei dispositivi di autovalutazione attivati durante I'anno. La seconda domanda è composta da due parti: una prima parte, a risposta chiusa, il cui intento è raccogliere un giudizio sintetico riguardo al nuovo metodo di valutazione utilizzato; una seconda parte, a risposta aperta, di motivazione del giudizio appena espresso.

Entrambi i questionari somministrati vengono compilati in forma anonima.

\section{Il secondo anno di sperimentazione (a.s. 2018/19)}

Durante l'anno scolastico, si sono realizzate, in media, 6 prove scritte e 4 prove orali per ogni studente di ogni classe. Nelle prove orali, si è scelto di consegnare un foglio con alcuni esercizi da svolgere in autonomia sul quale venivano dichiarati tutti gli obiettivi di apprendimento dell'oggetto di valutazione. Per quanto riguarda le verifiche scritte invece si è preferito dichiarare gli obiettivi con almeno una settimana di anticipo rispetto al giorno della prova, così da fornire agli studenti la possibilità di prepararsi più a fondo essendo consapevoli di quali fossero gli argomenti oggetto della prova scritta. Al momento della restituzione, sia nella prova scritta che in quella orale, ogni studente ha ricevuto un foglietto con i livelli raggiunti e i consigli dell'insegnante relativi a ogni obiettivo richiesto.

II momento della restituzione era molto atteso: molti studenti aspettavano con ansia di ricevere il fogliettino con i livelli raggiunti e, tornati al posto, lo studiavano con attenzione e concentrazione senza badare troppo a quello dei compagni. II confronto dei livelli presi in ogni obiettivo è molto più complicato da fare rispetto al voto numerico unico e infatti questa tendenza a vedere la valutazione degli altri si è ridotta già dalle prime prove.

Nel primo quadrimestre, per non sovraccaricare il lavoro degli studenti, non è stato chiesto loro di compilare il file dell'autovalutazione presente su Google Drive per fare in modo che si abituassero a ragionare con il nuovo metodo di valutazione. Solamente nel secondo quadrimestre è stata richiesta la compilazione del file "Le mie prove".

È stato creato un foglio di calcolo per raccogliere e numerare tutti i livelli degli alunni, suddivisi per obiettivo; in questo modo si è riusciti ad automatizzare il calcolo delle medie necessario per la decisione del voto finale, basta inserire i livelli raggiunti da 
ogni studente di volta in volta in ciascuna prova (in Allegato 7 viene proposto l'esempio del risultato di questo processo).

Oltre all'inserimento dei livelli, anche la scrittura dei consigli è avvenuta al computer (esempi di livelli disponibili in Allegato 8).

In corso d'anno, infatti, è emerso che alcune vecchie prove scritte erano piuttosto sbilanciate: a volte c'era un'abbondanza di esercizi focalizzati solo su obiettivi per lo più di aritmetica e algebra, a discapito di geometria, mentre per altri la quantità di esercizi dedicati era piuttosto carente. È stata cambiata per questo motivo la modalità di stesura delle prove, prestando più attenzione agli obiettivi coinvolti e al loro equilibrio nelle richieste così da ridurre gli esercizi ripetitivi incentrati solamente su un obiettivo; inoltre, per facilitare la correzione delle prove scritte, si è deciso di inserire per ciascun esercizio non più di 4-5 obiettivi di apprendimento.

In corso d'opera è stata anche apportata una modifica alla modalità della prova orale. Prestando attenzione all'elenco degli obiettivi di apprendimento e ai livelli raggiunti dagli studenti, si riusciva a impostare l'interrogazione in modo più completo, senza il rischio di dimenticare di verificare alcune conoscenze e abilità.

Anche la correzione delle prove è cambiata: per decidere quale livello assegnare a ogni obiettivo, infatti, durante la correzione è stato necessario concentrarsi su ognuno di essi, presente solitamente in modo trasversale in più esercizi.

Questo metodo di valutazione ha modificato infine anche l'andamento del colloquio tra docente e genitori: generalmente si avevano a disposizione molte più informazioni circa lo stato dell'apprendimento dello studente, potendo descrivere in modo maggiormente approfondito la sua situazione, fornendo consigli ad hoc affinché ci potesse essere un miglioramento.

\subsection{Alcuni dati emersi dai questionari}

Al questionario di inizio anno hanno partecipato 56 studenti su 61, mentre a quello di fine anno sono stati 59 .

Di seguito vengono presentate le tabelle con le risposte alle 13 domande a risposta multipla e i quesiti aperti con le motivazioni degli studenti organizzate per tematiche dalle quali si può fare un confronto tra la raccolta dati di inizio anno e quella di fine anno (Tabelle 2, 3, 4 e 5)

Tabella 2

Affermazioni del

questionario riguardanti

il "voto numerico".
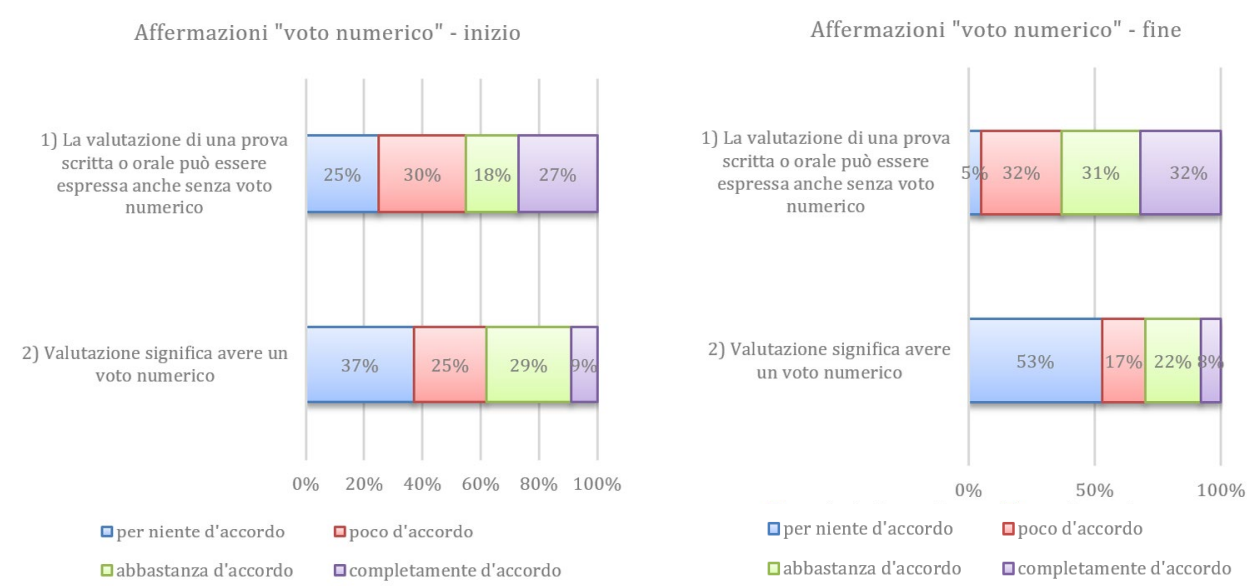
Valutare senza voto numerico: strumenti e riflessioni di una sperimentazione di matematica alle scuole secondarie di primo grado / Francesco D’Intino

Attraverso le prime due affermazioni volevo capire se i miei studenti associassero alla parola "valutazione" soltanto il voto numerico o se riuscissero a intenderla in maniera meno riduttiva.

Nell'affermazione 1) si nota che a fine anno circa il $20 \%$ degli studenti ha ampliato l'accezione della valutazione, non riducendola all'unica modalità più utilizzata, il voto numerico, ma accettando come strumento valutativo anche quello proposto nella sperimentazione.

Anche l'affermazione 2) conferma questo cambiamento, seppur in maniera meno evidente: gli studenti che non sono per niente d'accordo nel considerarsi valutati solo in presenza del voto numerico passano dal $37 \%$ al $53 \%$; si evidenzia un calo, di poco inferiore al $10 \%$, anche tra gli studenti che erano abbastanza d'accordo con I'affermazione, mentre quelli completamente d'accordo sono rimasti invariati.

Tabella 3

Affermazioni del

questionario riguardanti

il "feedback".
Affermazioni "feedback" - inizio

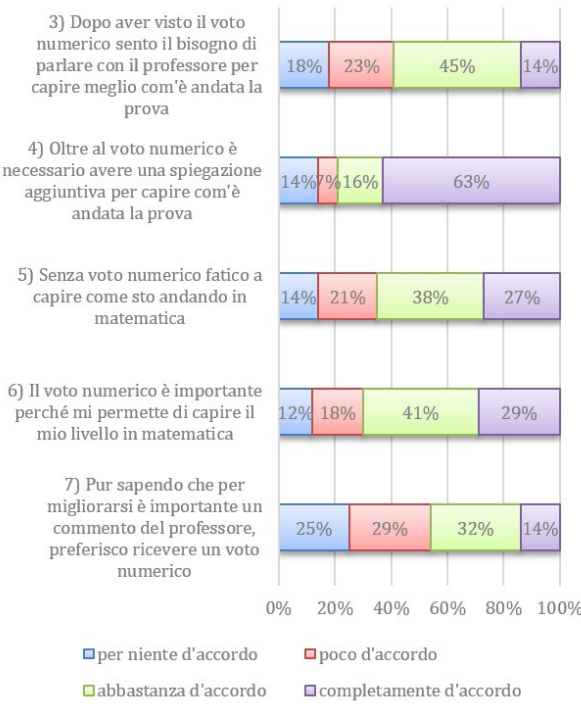

Affermazioni "feedback" - fine

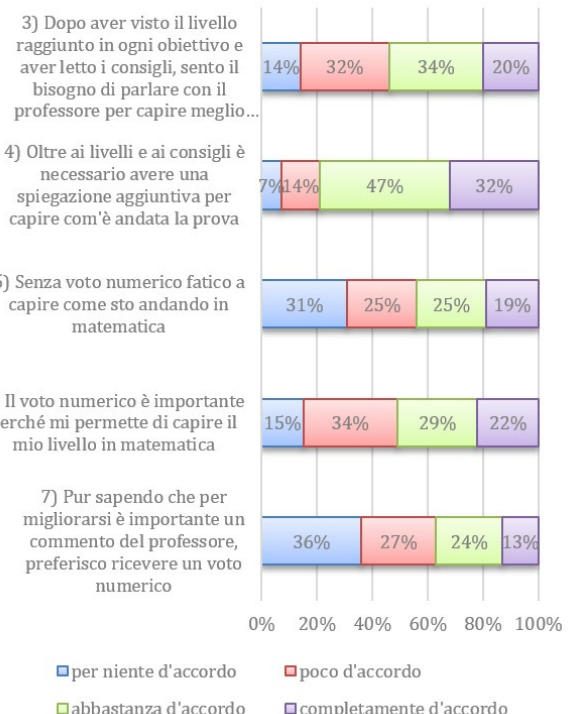

L'intento di queste cinque affermazioni era di comprendere se il nuovo metodo valutativo fosse stato utilizzato come strumento per comprendere l'andamento di una prova ed essere più consapevoli di ciò che si è appreso e ciò che invece necessita di un ulteriore impegno di studio.

Le affermazioni 3), 6) e 7) evidenziano un certo equilibrio nei questionari somministrati a inizio e a fine anno mentre nella 4) c'è uno spostamento significativo degli studenti che ritengono necessario avere una spiegazione aggiuntiva per capire il livello raggiunto: quelli completamente d'accordo si riducono dal $63 \%$ al $32 \%$, allo stesso modo si dimezzano anche quelli che si sono dichiarati per niente d'accordo, passando dal $14 \%$ al $7 \%$.

Anche nell'affermazione 5) ci sono importanti differenze tra le risposte di inizio e fine anno: gli studenti che sostenevano che il voto numerico fosse indispensabile per capire il loro livello in matematica sono diminuiti dal $65 \%$ al $44 \%$, a favore di quelli che ritengono il voto numerico del tutto inefficace per comprendere l'andamento di una prova che aumentano del $17 \%$. 
Tabella 4

Affermazioni riguardanti

il "come migliorarsi".
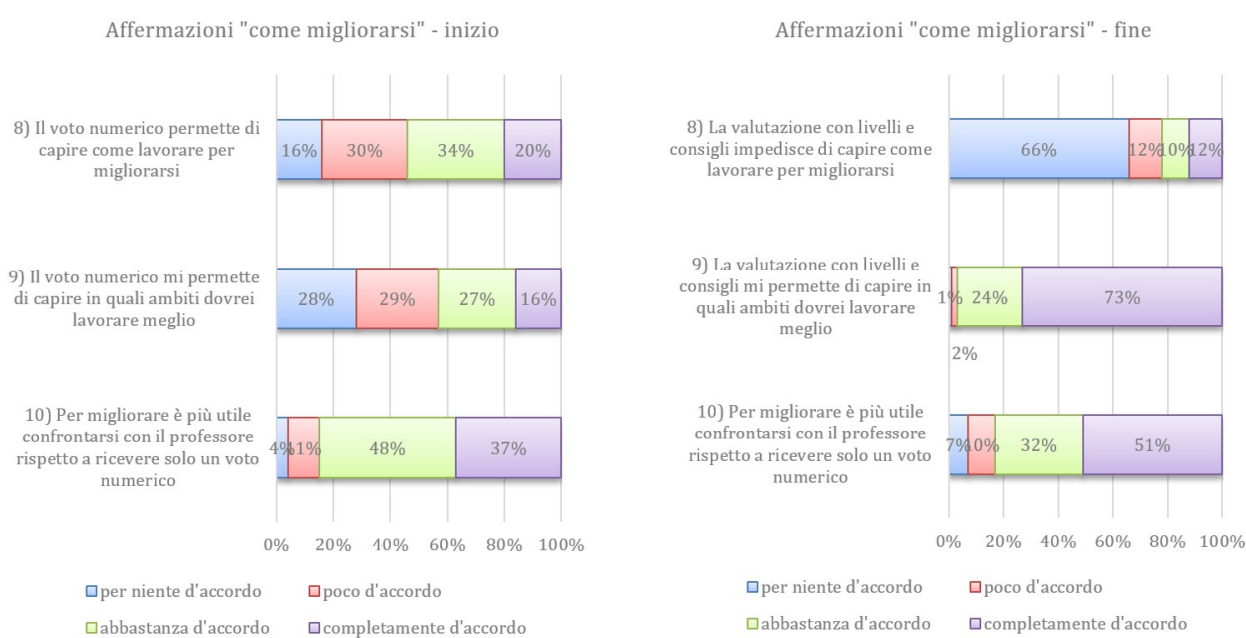

Le tre affermazioni "come migliorarsi" volevano analizzare l'incidenza della valutazione nel sostegno allo studio degli studenti, cioè se effettivamente questo metodo valutativo fornisce indicazioni più chiare su ciò che ognuno deve migliorare.

Se l'affermazione 10) non rileva particolari differenze, accade il contrario nella 8) e nella 9). In queste ultime il cambiamento è netto infatti nella 8) a inizio anno gli studenti che ritenevano il voto numerico utile per migliorare erano il $54 \%$ mentre a fine anno quelli che pensano lo stesso della valutazione a livelli aumentano fino al $78 \%$. L'affermazione 9) evidenzia dati ancora più netti, infatti se a inizio anno era il $43 \%$ degli studenti a pensare che il voto numerico aiutasse a capire in quali ambiti lavorare per migliorarsi, a fine anno gli studenti che ritengono fondamentale la valutazione a livelli per lo stesso scopo sono quasi la totalità (97\%).
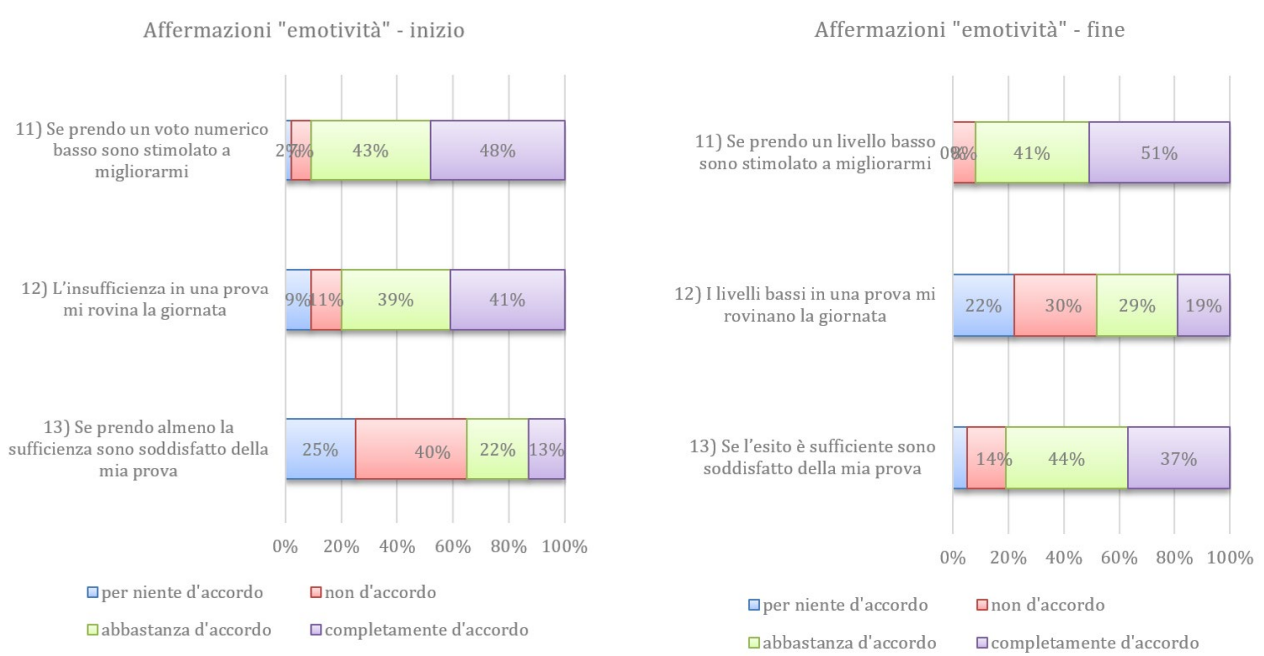

Le ultime tre affermazioni riguardano il coinvolgimento emotivo degli studenti rispetto ai vari metodi valutativi. Come si vede dall'affermazione 11), i risultati negativi, sia con il voto numerico che con i livelli, non sembrano intaccare la voglia di rivalsa degli studenti. Nell'affermazione 12) si nota come una prova non svolta bene abbia un minore impatto negativo a livello emotivo quando espressa con una valutazione a livelli (48\%) rispetto al voto numerico (80\%). Anche l'affermazione 13), riguardante la soddisfazione personale di una prova eseguita in modo positivo, mostra una per- 
Valutare senza voto numerico: strumenti e riflessioni di una sperimentazione di matematica alle scuole secondarie di primo grado / Francesco D’Intino

centuale maggiore di studenti gratificati nel caso dei livelli, 81\%, contro il 35\% della valutazione con voto numerico.

La seconda parte del questionario di fine anno comprendeva le due domande:

1. Quante volte hai completato il file "Le mie prove" su Google Drive (scrivendo il commento a caldo e la riflessione finale)?

2. Pensi che la modalità di valutazione con obiettivi, livelli e consigli ti abbia aiutato nello studio della matematica? Motiva la risposta in modo approfondito.

Di seguito viene presentata una breve analisi delle risposte.

Figura 2

Percentuale di risposte degli studenti alla prima

domanda del questionario di fine anno.

\section{1) Quante volte hai completato il file "Le mie prove" su Google Drive, scrivendo il commento a caldo e la riflessione finale?}

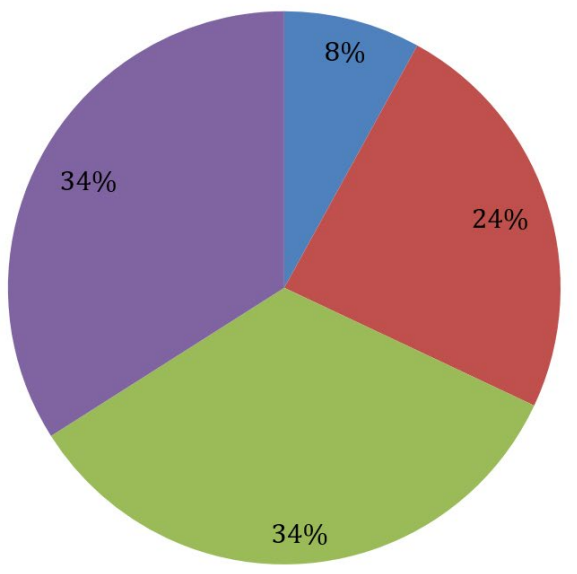

- Mai

Quasi mai

Quasi sempre

- Sempre

Come si nota dal grafico (Figura 2) c'è una maggioranza di studenti che ha compilato in maniera frequente il file dell'autovalutazione, $68 \%$, tuttavia rispetto all'anno precedente le differenze sono abbastanza contenute infatti per la stessa categoria la percentuale era del $62 \%$, cioè leggermente inferiore. Non avendo ancora trovato un modo veloce e sistematico per verificarne l'effettiva compilazione, non è stato possibile apprezzare un cambiamento più evidente tra i due anni scolastici.

Pensi che la modalità di valutazione con obiettivi, livelli e consigli ti abbia aiutato nello studio della matematica? Motiva la risposta in modo approfondito.

\section{Tabella 6}

Motivazioni relative alla risposta affermativa e negativa.

\begin{tabular}{|c|c|c|c|}
\hline sì & & NO & \\
\hline $\begin{array}{l}\text { Maggiore possibilità di capire in quali } \\
\text { ambiti lavorare di più }\end{array}$ & $37 \%$ & \multirow[t]{2}{*}{$\begin{array}{l}\text { Difficoltà nella comprensione della } \\
\text { valutazione }\end{array}$} & \multirow[t]{2}{*}{$10 \%$} \\
\hline $\begin{array}{l}\text { Migliore descrizione della situazione } \\
\text { dell'apprendimento }\end{array}$ & $28 \%$ & & \\
\hline $\begin{array}{l}\text { Maggiore concentrazione sugli errori } \\
\text { commessi e sulla correzione della } \\
\text { prova }\end{array}$ & $16 \%$ & \multirow[t]{2}{*}{ Metodo non sfruttato appieno } & \multirow[t]{2}{*}{$5 \%$} \\
\hline $\begin{array}{l}\text { Miglioramento dal punto di vista } \\
\text { emotivo }\end{array}$ & $4 \%$ & & \\
\hline & $85 \%$ & & $15 \%$ \\
\hline
\end{tabular}


Le risposte ricevute in questa domanda evidenziano che la gran parte degli studenti abbia percepito come utile il metodo valutativo proposto; infatti I' $85 \%$ degli studenti ha trovato giovamento nella valutazione con obiettivi, livelli e consigli; solo una piccola parte, circa il 15\%, sostiene di non essere stata aiutata dalla scelta. La maggioranza delle risposte positive (37\%) riguarda la possibilità di capire in quali ambiti della materia è necessario lavorare di più:

«Con i livelli riesco a capire meglio i miei errori e dove devo migliorare; questo metodo di valutazione lo approvo perché ti fa capire meglio gli errori mentre nel voto numerico non capisci benissimo i tuoi errori, soprattutto nelle interrogazioni»;

"Se sbaglio qualcosa con il voto numerico non saprei bene come ripassare, invece con i livelli sapendo più o meno come ripassare la volta dopo non ho fatto lo stesso errore»;

"Se ad esempio prendo un determinato voto in una verifica in cui ci sono più argomenti, non so in quali specifici argomenti devo migliorare e in quali va bene così, mentre con i vari livelli e consigli so su cosa devo soffermarmi»; "Se nel livello 32 ho preso 1, vado a vedere sul foglio e quello che c'è scritto me lo ristudio. Così quando nella prova successiva c'è il livello 32 sono ben preparato e se vado bene il professore mi potrebbe anche alzare il livello»; «Mi ha aiutata a capire dove dovevo migliorarmi e dove dovevo studiare di più ma anche le cose che non dovevo ripassare perché già le sapevo».

Una buona parte di studenti favorevoli al nuovo metodo (28\%) lo ritiene più completo perché descrive meglio la situazione del loro apprendimento:

«Con il voto numerico è difficile capire in cosa bisogna migliorare e dove si è bravi, perché è un voto per tutta la prova, invece con i livelli è possibile capire dove si è più forti e dove invece c'è da lavorare per ottenere livelli più alti nell'obiettivo in cui si è più deboli»;

«Mi ha permesso di capire gli argomenti in cui dovevo migliorare e adesso in vista dell'esame mi permetterà di capire quali argomenti che dovrò andare a riguardare meglio».

Un altro aspetto che emerge nel $16 \%$ delle motivazioni è che l'assenza del voto numerico fa sì che gli studenti non vengano più distratti da questo e che si concentrino sugli errori commessi e sulla correzione della prova:

«Dopo aver ricevuto la verifica con il foglietto degli obiettivi, certe volte quando vado a casa cerco di fare qualche esercizio simile a quello che c'è nella verifica e guardando le correzioni fatte sul quaderno ci riesco. Credo di essere migliorata molto rispetto all'inizio dell'anno grazie a questo metodo»;

«ll voto numerico mi faceva un po' guardare solamente il numero scritto in rosso anziché la prova, invece con questo metodo ho imparato e cercato di migliorare i miei punti deboli»;

«Quando vedo i miei livelli dopo una verifica riesco a capire e a risolvere subito il mio problema; poi non essendoci il voto numerico sto molto più attenta alle correzioni del professore». 
Anche a livello emotivo ci sono studenti (4\%) che evidenziano dei miglioramenti:

«Non mi sono scoraggiata o gasata vedendo i voti, ho solo controllato i livelli in cui non avevo preso tre o quattro e mi sono concentrata su quelli»;

«Se la prova non va molto bene non mi demoralizzo come accade con il voto numerico».

In qualche sporadica occasione, alcuni studenti hanno espresso il loro rammarico per I'assenza del voto numerico, nonostante fossero soddisfatti del metodo valutativo con obiettivi e livelli:

«La modalità con livelli e consigli mi ha aiutato a capire quali argomenti dovevo approfondire e rivedere, anche se non mi sarebbe dispiaciuto ricevere anche un voto numerico, anche solo come soddisfazione personale».

Le risposte negative sono decisamente meno e nella maggior parte (10\%) le motivazioni riguardano la difficoltà nella comprensione del metodo di valutazione, soprattutto per quanto riguarda i livelli raggiunti negli obiettivi i quali, secondo gli studenti, non riescono a risultare chiari nella descrizione dell'andamento della prova:

«Sono parecchio utili i consigli perché capisco dove devo migliorare ma non molto i livelli perché non so se sono andato bene o male»;

«ll metodo dei livelli l'ho trovato un po' complicato da capire»;

«I consigli sì, ma i livelli no perché non mi hanno dato proprio un'idea del mio andamento in matematica»;

«Mi sono sentito molto in difficoltà con lo studio, non capivo su che cosa andavo male e cosa andavo bene»;

«A me non mi ha aiutato perché non capisco che voto numerico ho»:

mentre in minima parte ( $5 \%$ ) alcuni studenti ammettono di non aver sfruttato appieno il metodo valutativo proposto, soprattutto perché si accontentavano di controllare esclusivamente l'esito della prova:

"Secondo me poteva aiutare molto sono io che non ho sfruttato come si deve l'occasione perché mi concentravo sempre sull'esito sufficiente o insufficiente altrimenti notando di più i miei sbagli nei livelli e nei consigli sarei potuto andare meglio capendo i miei errori»;

«In realtà io non ho fatto tanto caso ai livelli»;

«Sinceramente penso che se avessi sfruttato questa cosa dei livelli e dei consigli sarei riuscita ad andare meglio in matematica e mi avrebbe aiutato, l'errore è stato mio che non facevo niente per migliorare».

\section{Alcune riflessioni e conclusioni}

In questo contributo si è voluto presentare un'esperienza didattica, vissuta nell'arco di due anni scolastici, nella quale si sono sperimentati dispositivi valutativi incentrati 
in un'ottica principalmente formativa. Al termine di questi anni, è possibile effettuare alcune riflessioni e trarre alcune conclusioni, in termini di percezione degli allievi e dell'insegnante.

La motivazione per ideare e sperimentare un nuovo modello valutativo risiedeva principalmente nel fatto che molti studenti non si curavano della correzione delle verifiche perché erano più focalizzati nel fare confronti tra il loro voto e quello dei compagni. Da questo punto di vista, il metodo valutativo implementato ha avuto un certo effetto: la maggior parte degli allievi si concentra sui risultati ottenuti in termini di livelli di obiettivi. La correzione delle prove scritte è seguita con più attenzione; ogni studente sa che deve concentrarsi sugli esercizi in cui ha fatto errori e copiarli sul suo quaderno, consapevole degli obiettivi in cui ha ottenuto i livelli più bassi.

La griglia valutativa con gli indicatori, i consigli personalizzati per obiettivo e il rimando continuo agli aspetti importanti della materia hanno permesso agli studenti di avere una maggiore confidenza e conoscenza dei contenuti matematici in gioco. Anche per quanto riguarda la consapevolezza riguardo ai propri processi di apprendimento, i dispositivi pensati per l'autovalutazione hanno promosso un approccio metacognitivo che, per il momento, presenta ancora qualche problematica: la compilazione del file "Le mie prove" è ancora carente per la metà degli studenti; ciò può essere dovuto al fatto che non c'è un costante controllo da parte del docente, oppure alla poca abilità o familiarità con gli strumenti informatici o ancora alla percezione che sia poco utile. In futuro si potrebbe proporre l'acquisto di un quaderno specifico da destinare alla valutazione in matematica; su di esso dovranno essere raccolti i livelli raggiunti in ogni prova, i consigli ricevuti dall'insegnante e le riflessioni personali in ottica autovalutativa; per un controllo maggiore si potrebbe richiederne la consegna a ogni interrogazione e valutarne la compilazione nella prova orale, assegnando un livello alla partecipazione.

Al fine di promuovere sempre di più un atteggiamento positivo degli studenti verso I'attenzione ai contenuti e agli obiettivi su cui è necessario lavorare, si ipotizza nei prossimi anni di togliere dalla valutazione l'esito sintetico della prova (sufficiente/ insufficiente).

Questo metodo valutativo ha provocato visibili cambiamenti anche nella didattica, sia per quanto riguarda la progettazione sia per il controllo dell'apprendimento degli studenti. È emersa in corso d'opera una riflessione più profonda e consapevole rispetto a quali obiettivi verificare in ogni prova e anche su come equilibrarli nei vari esercizi scelti: in sostanza, aumenta la consapevolezza del docente di ciò che richiede agli studenti.

Questa progettazione più accurata richiede ovviamente un dispendio maggiore in termini di energie e di tempo. La correzione delle verifiche è infatti cambiata: dovendosi concentrare sui singoli obiettivi, la correzione passa da un esercizio all'altro focalizzando l'attenzione in modo trasversale su un obiettivo alla volta; anche la stesura dei consigli personalizzati deve essere curata perché è da questi che ogni studente troverà le indicazioni per migliorare il proprio apprendimento. La significativa mole di livelli assegnati per ogni obiettivo ha il pregio di indirizzare e guidare le scelte didattiche dell'insegnante, infatti da una breve analisi statistica si possono notare quali sono gli argomenti che hanno causato le maggiori difficoltà oppure quelli che sono stati appresi meglio. Di conseguenza, è possibile riprogettare la didattica prestando attenzione a queste informazioni. La gestione di tutti questi dati è possibile solamente se si possiede una discreta dimestichezza con i fogli di calcolo e l'automatizzazione di alcuni meccanismi di calcolo delle medie dei vari livelli; anche 
questo è un parametro da considerare se si vuole sperimentare questo metodo.

La scelta e la condivisione con gli studenti dei criteri di valutazione compiuta a inizio anno aiutano il docente a rimanere coerente con le scelte valutative stabilite durante il percorso scolastico e portano maggiore serenità: i dubbi nella decisione del voto da mettere a fine anno sono estremamente ridimensionati, perché la quantità di dati raccolti e la chiarezza nella modalità valutativa hanno fatto sì che non fosse necessario un ulteriore intervento sul voto finale né tantomeno una riflessione su come premiare l'impegno di qualche studente: tutto ciò era ben descritto dai criteri adottati e dalle informazioni raccolte durante l'anno.

La scelta di focalizzarsi sugli obiettivi ha lasciato un po' a margine i traguardi per lo sviluppo delle competenze; la valutazione per obiettivi richiede una tipologia di prove di verifica molto diversa da quella adatta alle competenze che è basata sui compiti di realtà. Nei prossimi anni si potrebbero sperimentare strategie valutative che includano anche i traguardi di competenze.

Una materia che probabilmente si presta maggiormente all'utilizzo dei traguardi per lo sviluppo delle competenze nella valutazione sono le scienze: in futuro sarebbe interessante predisporre una griglia valutativa anche per questa disciplina.

Un ultimo aspetto da approfondire ulteriormente nei prossimi anni è quello del coinvolgimento dei genitori degli studenti: si potrebbe ad esempio somministrare un questionario alle famiglie per indagare le loro convinzioni in materia valutativa e per conoscere il loro punto di vista riguardo al metodo sperimentato. Sarebbe interessante inoltre capire se la maggiore capacità descrittiva rispetto al voto numerico è apprezzata in quanto portatrice di maggiori informazioni oppure è causa di disagio e incomprensione.

L'idea è dunque quella di continuare a ideare, progettare e sollecitare tutti gli attori coinvolti nella valutazione, cercando di vivere e far vivere una scuola nella quale l'errore è visto come un'occasione di crescita e di comprensione perché, come afferma Sir John Enoch Powell, «L'unico vero errore è quello dal quale non impariamo nulla».

\section{Ringraziamenti}

Grazie a Michele Canducci, amico prima ancora che collega, senza il quale questo lavoro non avrebbe mai visto la luce.

Grazie a mia moglie Federica per il continuo sostegno, I'infinita pazienza e il prezioso confronto.

\section{Bibliografia}

Bacchi, S., \& Romagnoli, S. (2019). La classe senza voto. Torino: Loescher.

Bottero, E. (2014). La valutazione esterna degli apprendimenti scolastici. II caso delle prove INVALSI, Pedagogia più didattica, 1, 97-105.

Capperucci, D. (2011). La valutazione autentica nella scuola delle competenze e del successo formativo. In D. Capperucci (A cura di), La valutazione degli apprendimenti in ambito scolastico. Promuovere il successo formativo a partire dalla valutazione (pp. 63-100). Milano: Franco Angeli.

Castoldi, M. (2012). Valutare a scuola. Roma: Carocci. 
Valutare senza voto numerico: strumenti e riflessioni di una sperimentazione di matematica alle scuole secondarie di primo grado / Francesco D’Intino

Ciani, F. M. (2008). A scuola senza profitto. Legnago: Sempre Comunicazione.

Ciani, F. M. (2013). Scuola del gratuito. Legnago: Sempre Comunicazione.

Dozio, E. (2011). La valutazione degli allievi e la comunicazione ai genitori. Disponibile in https://docplayer.it/6359549-La-valutazione-degli-allievi-e-la-comunicazione-ai-genitori.html (consultato il 24.01.2020)

MIUR (2012). Indicazioni nazionali per il curricolo della scuola dell'infanzia e del primo ciclo di istruzione. Annali della Pubblica Istruzione, Numero Speciale. Le Monnier.

Tamagnini, D. (2019). Continuerò a sognarvi grandi. Milano: Longanesi.

Autore/Francesco D’Intino

Istituto Comprensivo Dante Alighieri - Rimini, Italia

dintino.francesco@icalighieri.it 